\title{
Toxicités relatives de divers pesticides pour Encarsia formosa Gahan (Hym., Aphelinidae) et pour son hôte, Trialeurodes vaporariorum Westw. (Hom., Aleyrodi- dae) *
}

Robert DELORME \& Andrée ANGOT

I.N.R.A. - Station de Phytopharmacie, Centre de Recherches agronomiques, Route de St-Cyr, F 78000 Versailles

RÉSUMÉ

La toxicité de 20 pesticides est étudiée sur le couple hôte-parasite Trialeurodes vaporariorum - Encarsia formosa. La méthode de laboratoire utilisée fait appel à des essais de longue durée, les produits étant appliqués sur tous les stades de l'hôte et du parasite. La plupart des fongicides testés sont utilisables en lutte intégrée, certains avec réserve (imazalil, triforine); seul le pyrazophos détruisant plus de $80 \mathrm{p} .100 \mathrm{des}$ populations d' $E$. formosa est à exclure formellement. Le cyhexatin, plus toxique pour l'hôte que pour le parasite, semble être un bon acaricide dans l'optique de la lutte intégrée. Tous les insecticides étudiés présentent une certaine toxicité pour $E$. formosa : la bioresméthrine et le dichlorvos, peu persistants, sont utilisables selon certaines modalités, de même que le pyrimicarbe; aphicide considéré comme spécifique qui est toxique pour les adultes du parasite. Les insecticides très persistants tels la deltaméthrine, le parathion éthyl et le pyrimiphos-méthyl sont absolument à proscrire (populations d' $E$. formosa réduites de plus de 95 p. 100).

L'étude des facteurs de toxicité de ces pesticides montre le danger de trop simplifier les méthodes d'appréciation de l'effet toxique des produits vis-à-vis des entomophages, des méthodes simples étant le plus souvent peu fiables.

L'accent est également mis sur la nécessité de raisonner en termes d'équilibre de population et non de toxicité absolue lorsqu'on a affaire comme ici à un entomophage lié spécifiquement à un hôte en milieu clos.

Mots clés additionnels : Lutte intégrée, Cultures protégées. Trialeurodes vaporarionum Westw. (Hom., Aleyrodidae).

The toxicity of 20 pesticides has been studied on the host-parasite couple Trialeurodes vaporariorum Encarsia formosa. Long-term trials were used under laboratory conditions. Pesticides were applied to the plant at all stages of host and parasite development.

Most of the tested fungicides could be used in integrated control, some of them with caution (imazalyl, triforine); only pyrazophos which kills more than $80 \%$ of $E$. formosa populations, must be excluded. Cyhexatin, which was more toxic for the host than for the parasite, seems to be a suitable miticide for integrated control. All insecticides studied showed some toxicity to $E$. formosa : poorly persistent products such as bioresmethrin and dichlorvos, ás well as pirimicarb, a specific aphicide, toxic to adult parasites, could be used with care. The highly persistent insecticides, like deltamethrin, ethyl-parathion and methylpirimiphos, must be excluded $(E$. formosa populations reduced more than $95 \%$ ).

When studying the toxicity factors of these pesticides, we observed that it is dangerous to simplify the methods for determining the toxic effects of these products to entomophagous insects. Simple methods are often insufficiently reliable.

We also emphasize the need to think in terms of balance of populations instead of absolute toxicity when the entomophagous insect under study is specifically host-dependent in glasshouse conditions.

Additional key words : Integrated control, Glasshouse crops. 


\section{INTRODUCTION}

Encarsia formosa Gahan est un hyménoptère de la famille des Aphelinidae. Il a été découvert en 1924, en GrandeBretagne, dans une serre où il parasitait la mouche blanche des serres, Trialeurodes vaporariorum Westw. (GAHAN, 1924). Très rapidement ses potentialités comme moyen biologique de lutte ont été démontrées et sa production à grande échelle a permis sa diffusion en Angleterre, au Canada, en Australie et Nouvelle-Zélande (ONILLON, 1982).

L'apparition des insecticides de synthèse voici une quarantaine d'années a progressivement conduit à l'abandon de cette méthode biologique de lutte : cette méthode séduisante, mais demandant une surveillance constante, a été remplacée par des traitements insecticides d'assurance beaucoup plus faciles a priori à mettre en ouvre.

Ainsi, pendant une trentaine d'années, la protection phytosanitaire des cultures sous abris est restée exclusivement chimique, avec l'utilisation constante d'une gamme de produits destinés à prévenir les principaux ravageurs (acariens, pucerons, aleurodes, thrips, ...) ou les principales maladies fongiques (Oïdium, Botrytis, ...). L'apparition de phénomènes de résistance chez les acariens et les aleurodes, la législation plus stricte concernant les résidus de pesticides ont conduit à reconsidérer le problème en proposant l'association de moyens de lutte biologique (Phytoseiulus persimilis A.H. contre Tetranychus urticae Koch et $E$. formosa contre $T$. vaporariorum) avec une protection phytosanitaire mieux conçue, utilisant si possible des produits plus spécifiques. Le but final est d'assurer une protection intégrée satisfaisante aussi bien du point de vue des coûts de production que sous l'angle des rendements et de la qualité des produits récoltés.

Une des principales difficultés rencontrées dans la définition d'un programme de lutte intégrée est la méconnaissance de l'effet des produits phytosanitaires envers les agents biologiques de lutte. L'expérience pratique a rapidement mis en évidence les problèmes posés par la coexistence des entomophages et l'application nécessaire de traitements destinés à lutter contre d'autres ravageurs ou contre les maladies fongiques. Des données résultant d'expérimentations au laboratoire ou en serre sont disponibles depuis une dizaine d'années. 'Citons chronologiquement : MAC ClANAHAN, 1970 ; IRVING \& WyATT, 1973 ; HELGESEN \& TAU. BER, 1974 ; ELENKOV et al., 1975 ; LEDIEU, 1979 ; KOWALSKA et al., 1980 ; IACOB et al., 1981.

Le but de cette étude est d'obtenir des données de laboratoire les plus complètes possibles en se rapprochant, dans des conditions standardisées, des structures de population telles qu'on les rencontre dans la pratique. On doit de cette sorte obtenir des données directement exploitables en culture protégée et mieux connaître les facteurs de toxicité des produits testés.

\section{MATÉRIEL ET MÉTHODE}

E. formosa est un endoparasite spécifique de $T$. vaporariorum. Les femelles, parthénogénétiques, pondent une soixantaine d'œufs dans les 3 derniers stades larvaires de l'hôte (Di PIETRO, 1977). Outre cette action parasitaire, on note également une action prédatrice au niveau des jeunes larves ( $1^{\text {er }}$ et $2^{\mathrm{e}}$ stades larvaires) (VAN ALPHEN et al., 1976). Les larves d'aleurodes parasitées noircissent après $12 \mathrm{j}$ environ à $22^{\circ} \mathrm{C}$ et l'émergence des adultes se produit environ $11 \mathrm{j}$ plus tard.
La méthode mise en cuvre ci-dessous tient compte de la biologie du parasite et des relations hôte-parasite, c'est-àdire que les effets des produits sont mesurés sur des associations des 2 insectes où sont présents les différents stades de chaque espèce. Les effets sont mesurés après un temps suffisamment long pour que tout effet toxique puisse en principe être mis en évidence.

Ces essais sont conduits sur tabacs (Nicotiana glauca Graham) plantés individuellement en pots recouverts par une housse de mousseline. La production des insectes, les infestations préalables et la conservation des plantes après traitement se font en chambre climatisée à $22 \pm 1{ }^{\circ} \mathrm{C}$, $70 \pm$ p. 100 d'HR et sous une photopériode de $16 \mathrm{~h}$, l'éclairage étant assuré par des tubes fluorescents.

Après avoir retiré les housses, le traitement se fait par pulvérisation d'une série de 5 solutions dont les concentrations en matière active forment une progression géométrique de raison 2 s'étageant de $1 / 8^{\mathrm{e}}$ au double de la concentration usuelle d'emploi (CE). La dose appliquée correspond à un dépôt moyen de $4 \mathrm{mg} / \mathrm{cm}^{2}$ de feuillage.

20 matières actives ont été essayées comprenant 13 fongicides, 1 acaricide spécifique et 6 insecticides (tabl. 1). Ce sont les produits les plus couramment utilisés en France en cultures légumières sous abri (le parathion éthyl est utilisé comme référence).

\section{A. Application du produit sur populations d'aleurodes non parasitées comportant tous les stades depuis l'œuf jusqu'à l'adulte (effet sur l'hôte) (Série A, fig. 1)}

Dans la mesure où l'emploi d'E. formosa, parasite spécifique de $T$. vaporariorum, est limité aux cultures protégées, il est très important de connaître la toxicité des produits testés pour l'hôte non parasité dans le but, d'une part, d'interpréter les éventuelles toxicités pour les stades internes du parasite (toxicité directe du produit pour la larve ou la nymphe du parasite présent à l'intérieur des larves d'aleurodes, ou mortalité indirecte par mort de l'hôte) et, d'autre part, d'apprécier l'effet global d'un traitement sur les équilibres de populations.

Les plants de tabac sont contaminés $25 \mathrm{j}$. avant le traitement par 20 aleurodes adultes et de nouveau $12 \mathrm{j}$. plus tard de telle sorte qu'au moment du traitement la plante porte des populations d'aleurodes comportant des adultes d'âge variable et tous les autres stades depuis l'œuf fraîchement pondu jusqu'à l'adulte prêt à émerger du puparium.

L'effet du traitement est vérifié $28 \mathrm{j}$. plus tard, c'est-àdire le temps d'une génération, en contrôlant sur les plantes le nombre de pupariums éclos. On effectue ensuite une comparaison des plantes traitées au témoin, le résultat se traduisant par une réduction de population par rapport au témoin.

\section{B. Applications du produit sur les stades pré-imaginaux} d'E. formosa (séries B et C, fig. 1)

Deux séries complémentaires sont réalisées :

1. Application sur jeunes stades du parasite (avant noircissement des larves d'aleurodes parasitées (série B).

$28 \mathrm{j}$. avant le traitement, les tabacs sont contaminés par 50 aleurodes adultes $; 17 \mathrm{j}$. plus tard, temps nécessaire pour que les larves soient réceptives pour le parasite, 25 adultes d'E. formosa sont apportés et laissés pendant $7 \mathrm{j}$. Au moment du traitement, les plantes portent donc des aleurodes de tous stades parasités ou non, $E$. formosa étant 
TABLEAU 1

Liste des produits phytosanitaires testés.

List of tested pesticides.

\begin{tabular}{|c|c|c|c|c|}
\hline $\begin{array}{l}\text { Matière } \\
\text { active }\end{array}$ & $\begin{array}{l}\text { Produit } \\
\text { commercial }\end{array}$ & $\begin{array}{l}\text { Teneur } \\
\text { en matière } \\
\text { active } \\
\text { (M.A.) }\end{array}$ & $\begin{array}{l}\text { Concentration } \\
\text { d'emploi en } \\
\text { g M.A./hl } \\
\text { (C.E.) }\end{array}$ & Usage \\
\hline
\end{tabular}

\section{FONGICIDES}

\begin{tabular}{|c|c|c|c|c|}
\hline bénomyl & Benlate & $50 \%$ & 50 & Botrytis, cercosporiose \\
\hline bupirimate & Nimrod & $250 \mathrm{~g} / 1$ & 25 & Oidium \\
\hline captafol & Orthodifolatan $80 \mathrm{PM}$ & $80 \%$ & 160 & mildiou, Alternaria \\
\hline chinométhionate & Morestan & $25 \%$ & 12,5 & Oidium, tétranyque \\
\hline dichlofluanide & Euparène & $50 \%$ & 125 & Botrytis, mildiou, Oïdium \\
\hline folpel & Mollona & $50 \%$ & 150 & $\begin{array}{l}\text { mildiou, excoriose, anthracnose, } \\
\text { septoriose }\end{array}$ \\
\hline imazalil & Fungaflor & $200 \mathrm{~g} / 1$ & 40 & Oïdium \\
\hline iprodione & Rovral & $50 \%$ & 75 & Botrytis, Sclerotinia \\
\hline mancozèbe & Dithane M 45 & $80 \%$ & 160 & mildiou, Alternaria, anthracnose... \\
\hline pyrazophos & Afugan & $295 \mathrm{~g} / 1$ & 15 & Oidium \\
\hline thirame & Thiramac & $80 \%$ & 350 & Botrytis, anthracnose, Coryneum \\
\hline triforine & Saprol & $190 \mathrm{~g} / 1$ & 28,5 & Ö̈dium, cladosporiose \\
\hline vinchlozoline & Ronilan & $50 \%$ & 100 & Botrytis, Sclerotinia \\
\hline \multicolumn{5}{|c|}{ ACARICIDE SPÉCIFIQUE } \\
\hline cyhexatin & Plictran & $25 \%$ & 30 & tétranyques \\
\hline \multicolumn{5}{|l|}{ INSECTICIDES } \\
\hline bioresmethrine & Isathrine & $100 \mathrm{~g} / \mathrm{l}$ & 6 & aleurodes \\
\hline deltaméthrine & Décis & $25 \mathrm{~g} / 1$ & 1,25 & $\begin{array}{l}\text { aleurodes, pucerons, lépidoptères, } \\
\text { (mineuses) }\end{array}$ \\
\hline dichlorvos & Nogos 50 ĚV & $500 \mathrm{~g} / 1$ & 100 & $\begin{array}{l}\text { pucerons, thrips, aleurodes, } \\
\text { acariens }\end{array}$ \\
\hline parathion-éthyl & Rhodiatox & $100 \mathrm{~g} / 1$ & 25 & insecticide, polyvalent (référence) \\
\hline pyrimicarbe & Pirimor & $50 \%$ & 37,5 & pucerons \\
\hline pyrimiphos-méthyl & PP 511 & $250 \mathrm{~g} / \mathrm{l}$ & 50 & aleurodes, pucerons \\
\hline
\end{tabular}

présent au stade cuf et jeune larve avant noircissement de la larve d'aleurode.

Un $1^{\text {cr }}$ contrôle est effectué $24 \mathrm{j}$. après traitement et l'on compare les émergences d'adultes d' $E$. formosa dans les lots traités et témoins; un $2^{\mathrm{e}}$ contrôle a lieu $35 \mathrm{j}$. après le $1^{\mathrm{er}}$ et l'on mesure alors le pourcentage de parasitisme au niveau de la génération issue des $E$. formosa traités.

2. Application sur stades âgés du parasite (stades internes aux pupariums noirs d'aleurodes) (série C).

Cette série est semblable à la précédente dans son principe ; seules les périodes de contamination sont différentes ; elles sont étudiées de telle façon qu'au moment du traitement les plantes portent principalement des $E$. formosa au stade « pupes noires » et des larves d'aleurodes non parasitées. Un $1^{\text {er }}$ contrôle est effectué $14 \mathrm{j}$ après traitement et l'on note pour chaque plante le pourcentage d'éclosion des adultes d' $E$. formosa à partir des pupariums noirs. Un $2^{\circ}$ contrôle est effectué identiquiement à la série précédente.

\section{Application du produit sur plantes et étude pour les parasites adultes de la toxicité initiale et de la toxicité résiduelle des dépôts ainsi formés (série $D$, fig. 1)}

$15 \mathrm{j}$. avant traitement, les plantes sont contaminées par 15 aleurodes adultes; elles sont ainsi plus attractives pour $E$. formosa et des larves de l'hôte assurent la nourriture des parasites (succion du miellat émis et prédation). Après traitement, 50 à 100 parasites adultes, par concentration testée, sont apportés le jour de l'intervention, puis après 1 , $2,6,9,15 \mathrm{j}$. et, éventuellement, chaque semaine ultérieure. Les parasites âgés de 0 à $1 \mathrm{j}$. sont prélevés dans l'élevage et leurs mortalités sont relevées après $24 \mathrm{~h}$ de contact.

Les différentes opérations réalisées pour ces 4 séries sont schématisées dans la figure 1.

Un aspect important de la toxicité des pesticides pour $E$. formosa est difficilement mesurable avec précision sur plantes entières: il s'agit de la toxicité directe pour les adultes présents lors du traitement ; un essai complémentaire a été réalisé dans ce but. 


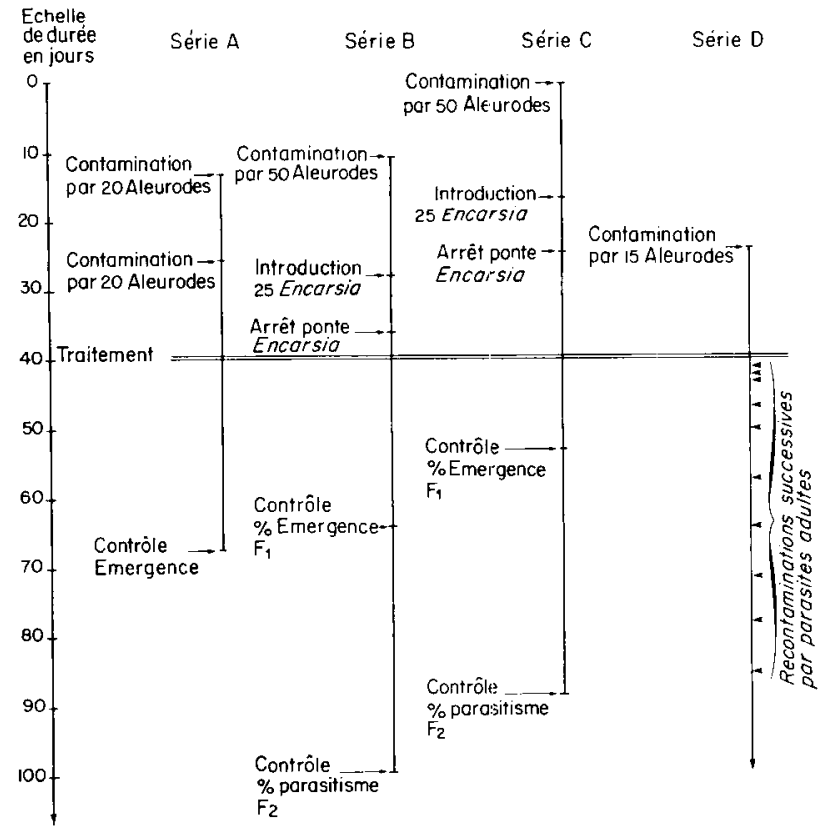

Figure 1

Schéma général de l'essai.

General scheme of the trial.

\section{Application directe du produit sur adultes du parasite}

Les adultes, après immobilisation par le froid, sont traités à l'aide d'une tour de pulvérisation permettant d'obtenir des dépôts reproductibles de $4 \mathrm{mg} / \mathrm{cm}^{2}$ sur des disques foliaires. Les mortalités sont relevées après $24 \mathrm{~h}$. Les essais répétés au moins 2 fois conduisent à déterminer la CL 50 de chaque produit et permettent ainsi de caractériser le risque de toxicité pour les adultes présents lors des traitements.

\section{RÉSULTATS}

\section{A. Action des produits sur populations d'aleurodes non parasitées (série A)}

La toxicité du produit est exprimée par la réduction d'émergence des adultes par rapport au témoin. Les chiffres obtenus permettent de déterminer une droite de régression - $\log$ des concentrations, probits des pourcentages de réduction - à partir de laquelle sont calculés les résultats mentionnés dans les tableaux 2 et suivants pour les concentrations d'emploi $(\mathrm{CE})$, double $(2 \mathrm{CE})$ et moitié $\frac{\mathrm{CE} \text {. }}{2}$

A la concentration pratique d'emploi, parmi les fongicides, seuls le chinométhionate, le mancozèbe et, à un degré moindre, la triforine présentent une certaine toxicité pour les aleurodes; cette toxicité est inférieure à celle des insecticides et acaricides à l'exception du pyrimicarbe pour lequel, dans les conditions de l'essai, aucun effet n'a pu être mis en évidence. La majorité des fongicides et le pyrimicarbe étant sans action sur l'hôte, une toxicité du produit considéré, même faible, vis-à-vis d' $E$. formosa pourra jouer défavorablement sur l'équilibre hôte-parasite.
TABLEAU 2

Réduction des populations d'aleurodes observées par rapport au témoin.

Reduction of whitefly populations compared to the control.

\begin{tabular}{|c|c|c|c|}
\hline \multirow[b]{2}{*}{ Matic̀res actives } & \multicolumn{3}{|c|}{$\begin{array}{l}\text { Pourcentages de réduction } \\
\text { aux concentrations } \\
\text { indiquées }\end{array}$} \\
\hline & $2 \mathrm{CE}$ & $\mathrm{CE}$ & $\frac{\mathrm{CE}}{2}$ \\
\hline bénomyl & 10,2 & 3,9 & 1,2 \\
\hline bupirimate & 0 & 0 & 0 \\
\hline captafol & 9,0 & 0,1 & 0 \\
\hline chinométhionate & 63,8 & 54,6 & 45,2 \\
\hline dichlofluanide & 0 & 0 & 0 \\
\hline folpel & 1,1 & 0,2 & 0,1 \\
\hline imazalil & 8,8 & 0,1 & 0 \\
\hline iprodione & 11,7 & 8,0 & 5,3 \\
\hline mancozèbe & 51,5 & 43,2 & 35,1 \\
\hline pyrazophos & 10,5 & 6,1 & 3,3 \\
\hline thirame & 0 & 0 & 0 \\
\hline triforine & 32,0 & 24,5 & 18,1 \\
\hline vinchlozoline & 0 & 0 & 0 \\
\hline cyhexatin & 73,8 & 64,3 & 53,7 \\
\hline bioresméthrine & 81,1 & 60,1 & 35,8 \\
\hline deltaméthrine & 95,7 & 92,7 & 88,5 \\
\hline dichlorvos & 67,1 & 62,7 & 58,0 \\
\hline parathion-éthyl & 91,5 & 86,7 & 80,4 \\
\hline pyrimicarbe & 0 & 0 & 0 \\
\hline pyrimiphos-méthyl & 78,1 & 66,5 & 53,1 \\
\hline
\end{tabular}

$\mathrm{CE}=$ concentration d'emploi.

\section{B. Action des produits sur les stades pré-imaginaux d'E. formosa (séries B et C)}

Pour la série B (stades internes aux larves d'aleurodes avant noircissement), l'effet est mesuré par la réduction d'émergence des parasites adultes par rapport au témoin, alors que pour la série $C$ (stades internes aux pupariums noirs d'aleurodes) la toxicité est appréciée par le pourcentage de pupariums noirs non éclos corrigé par celui des témoins. Les résultats sont donnés dans le tableau 3.

Parmi les 13 fongicides testés, seuls le chinométhionate, le pyrazophos et la triforine ont une action sur les stades préimaginaux d'E. formosa. Le pyrimicarbe est le seul insecticide à n'avoir aucune action.

Dans pratiquement tous les cas, les jeunes stades du parasite sont plus sensibles que les stades plus âgés, le puparium noir de l'hôte devant offrir une certaine protection vis-à-vis des produits. Les 2 exceptions, deltaméthrine et pyrimiphos méthyl qui présentent l'effet inverse sont parmi les produits les plus persistants: de fait, à l'émergence, lors de la confection du trou de sortie, les adultes ont été tués par les résidus de produits présents à la surface du puparium et n'ont donc pas pu éclore normalement.

Les réductions d'émergences des populations traitées aux stades jeunes sont probablement dues en grande partie à la toxicité des produits envers l'hôte, la mort de celui-ci entraînant la mort de l'endoparasite. La seule exception semble être le pyrazophos qui, n'ayant qu'une très faible action sur l'hôte, réduit néanmoins fortement les émergences d'E. formosa ; il présente donc une forte toxicité sélective pour le parasite. 
TABLEAU 3

Action des produits sur les stades pré-imaginaux d'Encarsia formosa.

Toxicity of pesticides to pre-imaginal stages of Encarsia formosa.

\begin{tabular}{|c|c|c|c|c|c|c|}
\hline \multirow[b]{2}{*}{ Matières actives } & \multicolumn{3}{|c|}{ Stades jeunes $\left({ }^{*}\right)$} & \multicolumn{3}{|c|}{ Stades âgés $\left({ }^{* *}\right)$} \\
\hline & $2 \mathrm{CE}$ & $\mathrm{CE}$ & $\frac{\mathrm{CE}}{2}$ & $2 \mathrm{CE}$ & $\mathrm{CE}$ & $\frac{\mathrm{CE}}{2}$ \\
\hline bénomyl & 0 & 0 & 0 & 0 & 0 & 0 \\
\hline bupirimate & 0 & 0 & 0 & 0 & 0 & 0 \\
\hline captafol & 0 & 0 & 0 & 0 & 0 & 0 \\
\hline chinométhionate & 30,1 & 28,5 & 26,9 & 3,3 & 1,7 & 0,3 \\
\hline dichlofluanide & 0 & 0 & 0 & 0 & 0 & 0 \\
\hline folpel & 0 & 0 & 0 & 0 & 0 & 0 \\
\hline imazalil & 2,0 & 0,1 & 0 & 5,2 & 0,1 & 0 \\
\hline iprodione & 0 & 0 & 0 & 0 & 0 & 0 \\
\hline mancozèbe & 0 & 0 & 0 & 0 & 0 & 0 \\
\hline pyrazophos & 76,0 & 74,8 & 73,6 & 71,5 & 39,1 & 13,0 \\
\hline thirame & 0 & 0 & 0 & 0 & 0 & 0 \\
\hline triforine & 77,8 & 72,9 & 67,5 & 35,8 & 29,6 & 24,0 \\
\hline vinchlozoline & 0 & 0 & 0 & 0 & 0 & 0 \\
\hline cyhexatin & 23,5 & 11,1 & 4,3 & 0,7 & 0 & 0 \\
\hline bioresméthrine & 80,6 & 69,6 & 56,4 & 10,6 & 4,0 & 1,2 \\
\hline deltaméthrine & 91,9 & 70,1 & 36,7 & 100 & 99,9 & 98,7 \\
\hline dichlorvos & 25,3 & 21,9 & 18,7 & 14,9 & 0,1 & 0 \\
\hline parathion-éthyl & 100 & 99,9 & 99,1 & 98,4 & 93,4 & 80,6 \\
\hline pyrimicarbe & 0 & 0 & 0 & 0 & 0 & 0 \\
\hline pyrimiphos-méthyl & 93,6 & 87,8 & 79,1 & 99,6 & 97,8 & 91,8 \\
\hline
\end{tabular}

$\mathrm{CE}=$ concentration d'emploi.

$\left(^{*}\right)$ Stades vivant dans les larves d'aleurodes : toxicité appréciée par le pourcentage de réduction des éclosions imaginales.

$\left.{ }^{* *}\right)$ Stades vivant dans les pupariums d'aleurodes : toxicité appréciée par le pourcentage de pupariums noirs non éclos.

TABLEAU 4

Action des produits sur les adultes d'E. formosa.

Toxicity of pesticides to $\mathrm{E}$. formosa adults.

\begin{tabular}{|c|c|c|c|c|c|c|c|c|c|c|c|}
\hline \multirow[b]{3}{*}{ Matières actives } & \multicolumn{6}{|c|}{ Toxicité initiale et persistance d'action des résidus } & \multicolumn{5}{|c|}{ Toxicité directe des produits } \\
\hline & \multicolumn{3}{|c|}{$\begin{array}{c}\text { Toxicité initiale } \\
\text { (pourcentage } \\
\text { mortalité) }\end{array}$} & \multicolumn{3}{|c|}{$\begin{array}{l}\text { Persistance d'action } \\
\text { des résidus }\left({ }^{*}\right)\end{array}$} & \multicolumn{3}{|c|}{ \% mortalité } & \multirow[t]{2}{*}{$\begin{array}{l}\text { CL } 50 \text { en } \\
\text { g MA } / \mathrm{hl}\end{array}$} & \multirow{2}{*}{$\frac{\begin{array}{c}\text { Rapport } \\
\text { CL } 50\end{array}}{\mathrm{CE}}$} \\
\hline & $2 \mathrm{CE}$ & CE & $\frac{C E}{2}$ & $2 \mathrm{CE}$ & $\mathrm{CE}$ & $\frac{\mathrm{CE}}{2}$ & $2 \mathrm{CE}$ & $\mathrm{CE}$ & $\frac{\mathrm{CE}}{2}$ & & \\
\hline bénomyl & 0,2 & 0 & 0 & 0 & 0 & 0 & 0,2 & 0 & 0 & 1468 & 29,4 \\
\hline bupirimate & 0,8 & 0 & 0 & 0 & 0 & 0 & 0,8 & 0 & 0 & 390 & 15,6 \\
\hline captafol & 0 & 0 & 0 & 0 & 0 & 0 & 0 & 0 & 0 & 4700 & 29,4 \\
\hline chinométhionate & 0 & 0 & 0 & 0 & 0 & 0 & 28,3 & 15,6 & 7,4 & 62,11 & 2,48 \\
\hline dichlofluanide & 0 & 0 & 0 & 0 & 0 & 0 & 1,4 & 0,3 & 0,1 & 3342 & 26,6 \\
\hline folpel & 0 & 0 & 0 & 0 & 0 & 0 & 0 & 0 & 0 & 3762 & 25,0 \\
\hline imazalil & 97,9 & 93,6 & 80,1 & 4,7 & 3,7 & 2,9 & 98,6 & 93,6 & 80,1 & 8,401 & 0,420 \\
\hline iprodione & 0 & 0 & 0 & 0 & 0 & 0 & 0 & 0 & 0 & 2525 & 33,7 \\
\hline mancozèbe & 3,5 & 0 & 0 & 0 & 0 & 0 & 22,8 & 14,9 & 9,0 & 1823 & 11,4 \\
\hline pyrazophos & 99,6 & 78,0 & 8,5 & $>100$ & 69,4 & 0 & 100 & 100 & 99,2 & 1,151 & 0,0767 \\
\hline thirame & 0 & 0 & 0 & 0 & 0 & 0 & 7,7 & 1,9 & 0,3 & 3138 & 8,97 \\
\hline triforine & 3,0 & 3,0 & 2,2 & 0 & 0 & 0 & 22,6 & 8,4 & 2,2 & 131,2 & 4,60 \\
\hline vinchlozoline & 0 & 0 & 0 & 0 & 0 & 0 & 4,1 & 1,0 & 0,2 & 1700 & 17,0 \\
\hline cyhexatin & 45,4 & 10,6 & 6,6 & 5,3 & 0 & 0 & 45,4 & 20,9 & 6,6 & 67,29 & 2,24 \\
\hline bioresméthrine & 69,9 & 56,7 & 42,8 & 20,0 & 14,4 & 7,4 & 100 & 100 & 100 & 0,1630 & 0,0272 \\
\hline deltaméthrine & 99,9 & 99,6 & 95,3 & $>100$ & 96,3 & 49,1 & 100 & 100 & 100 & 0,005418 & 0,00433 \\
\hline dichlorvos & 100 & 95,3 & 95,3 & 18,4 & 8,9 & 7,9 & 100 & 100 & 100 & 0,1375 & 0,00137 \\
\hline parathion-éthyl & 100 & 100 & 100 & $>100$ & $>100$ & 63,8 & 100 & 100 & 100 & 0,03840 & 0,00154 \\
\hline pyrimicarbe & 99,9 & 99,3 & 82,1 & 9,8 & 8,8 & 8,8 & 99,8 & 98,5 & 92,7 & 4,621 & 0,123 \\
\hline pyrimiphos-méthyl & 100 & 100 & 100 & $>100$ & 66,6 & 33,3 & 100 & 100 & 100 & 0,06356 & 0,00127 \\
\hline
\end{tabular}

${ }^{*}$ ) Nombre de jours au bout duquel les résidus ne provoquent plus que 20 p. 100 de mortalité. $\mathrm{CE}=$ concentration d'emploi. 
Le cas du mancozèbe est également à considérer : bien que réduisant fortement les populations d'aleurodes il n'a aucun effet sur $E$. formosa. Des essais complémentaires ont montré que, parmi les stades de $T$. vaporariorum, les seuls sensibles sont les stades L1-L2 donc trop jeunes pour accueillir le parasite, tandis que les stades réceptifs (L3-L4) ne sont pratiquement pas touchés par le mancozèbe.

\section{Toxicité initiale et résiduelle des dépôts formés sur les plantes, pour les adultes d'E. formosa}

Les résultats sont présentés dans le tableau 4. Seuls l'imazalil et le pyrazophos, parmi les fongicides testés, présentent une importante toxicité pour les adultes venant fréquenter les plantes traitées. Si l'effet toxique du premier disparaît rapidement $(3$ à $4 \mathrm{j}$ pour la concentration d'emploi), le pyrazophos fait preuve d'une persistance d'action comparable à celle d'insecticides non sélectifs (supérieure à 2 mois dans les conditions du laboratoire) semblant incompatible avec une lutte biologique efficace.

La plupart des insecticides-acaricides font preuve d'une forte toxicité initiale (mortalité supérieure à 95 p. 100), le cyhexatin, acaricide spécifique, et la bioresméthrine faisant exception ; ils se répartissent en 2 groupes lorsqu'on regarde leur persistance d'action : celle-ci est faible pour la bioresméthrine, le dichlorvos et le pyrimicarbe (inférieure à $15 \mathrm{j}$ ) elle est, très importante par contre pour la deltaméthrine, le parathion-éthyl et le pyrimiphos-méthyl (supérieure à 2 mois).

\section{Toxicité des produits appliqués en pulvérisation sur les adultes d' $E$. formosa.}

Ces résultats sont dans l'ensemble comparables aux toxicités initiales relevées sur plantes (tabl. 4). En effet, comme dans le cas précédent, tous les insecticides, l'imazalil et le pyrazophos appliqués à leurs concentrations d'emploi respectives provoquent plus de 90 p. 100 de mortalité ; tous les autres produits donnant des mortalités voisines ou inférieures à 20 p. 100 .

Le rapport concentration létale 50/concentration d'emploi $\frac{C L 50}{C E}$ permet de préciser le risque de toxicité directe des différents produits ; en effet le pourcentage de mortalité relevé à la concentration pratique d'emploi n'est qu'indicatif : les conditions climatiques (température et hygrométrie), la quantité de liquide appliquée sont autant de facteurs qui peuvent modifier dans un sens ou dans l'autre l'intensité de la réponse. On peut à l'aide de ce rapport essayer de préciser ces risques en prenant le barème suivant :

forte toxicité : rapport $<1$

risque important de toxicité : $5>$ rapport $>1$

risque de toxicité faible ou nul : rapport $>5$.

Dans la $1^{\text {re }}$ catégorie on trouve tous les insecticides, l'imazalil et le pyrazophos. Notons la très forte toxicité directe de la deltaméthrine, du dichlorvos, du parathion éthyl et du pyrimiphos méthyl (CL. 50 de 100 à 1000 fois plus faible que la $\mathrm{CE}$ ).

Le chinométhionate, la triforine et le cyhexatin peuvent, dans certaines conditions, se révéler dangereux pour les adultes présents lors du traitement. Les autres produits, tous fongicides, peuvent très probablement être utilisés sans risque.

\section{E. Incidence des traitements sur le parasitisme obtenu au niveau de la $2^{*}$ génération}

L'examen du pourcentage de parasitisme obtenu au niveau de la génération issue de la génération traitée n'a pas toujours permis la mise en évidence de différences entre les plantes traitées et les plantes témoins : seuls la deltaméthrine, le parathion-méthyl et le pyrimiphos-méthyl réduisent de façon importante le parasitisme, totalement pour les 2 premiers et de plus de 90 p. 100 pour le dernier.

Il semble que l'on aurait dû également observer sur le parasitisme une action résultant des traitements avec d'autres produits qui se sont révélés toxiques dans les essais précédents. En fait, les effets ont été probablement masqués par plusieurs facteurs: la méthode utilise un fort parasitisme au niveau de la $1^{\text {rc }}$ génération et même si l'on réduit de façon importante les émergences ou la survie des parasites adultes, le nombre d'hôtes disponibles pour la $2^{\mathrm{e}}$ génération est probablement trop faible pour que l'on puisse mettre en évidence autre chose que des destructions presque totales des populations du parasite, telles celles produites par les 3 insecticides ci-dessus; de plus, dans ces essais, la toxicité directe du produit pour les adultes présents lors du traitement n'est pas prise en compte.

Devant cette impossibilité de mettre en évidence directement l'impact global d'un traitement sur l'équilibre hôteparasite, nous avons établi une valeur théorique calculée, intégrant tous les facteurs de toxicité pour les différentes composantes de la population d'E. formosa, appelée «toxicité pour les populations d'E. formosa ".

\section{F. Toxicité pour les populations d'E. formosa}

Compte tenu de la durée de développement d'E. formosa et de la longévité des adultes, on peut estimer qu'au moment d'un traitement en serre la population totale est constituée d'environ $2 / 3$ de stades préimaginaux et de $1 / 3$ d'adultes. L'impact du traitement sur les stades préimaginaux est apprécié par la notion de réduction de population de $E$. formosa ayant émergé et qui est restée vivante $24 \mathrm{~h}$ après l'émergence (intégration de la persistance d'action), la toxicité pour les adultes étant connue par l'expérimentation directe.

Les résultats ainsi obtenus sont donnés dans le tableau 5 et comparés aux toxicités pour $T$. vaporariorum. On peut de cette façon déterminer les incidences favorables ou défavorables des traitements sur l'équilibre hôte-parasite.

Les applications de bénomyl, bupirimate, captafol, dichlofluanide, folpel, iprodione, thirame et vinchlozoline n'entraînent aucun effet notable aussi bien sur l'hôte que sur le parasite et semblent pouvoir être utilisés sans gros problème dans un programme de lutte intégrée faisant intervenir $E$. formosa. Parmi les insecticides, la biosresméthrine et le dichlorvos détruisent environ les $2 / 3$ des populations aussi bien de l'hôte que du parasite; ils devraient donc être sans grande incidence sur l'équilibre, cependant leur application doit être subordonnée à la composition des populations lors du traitement, les adultes d' $E$. formosa étant extrêmement sensibles à chacune de ces 2 molécules. Peuvent également être utilisés sans grand inconvénient, le chinométhionate, le mancozèbe et le cyhexatin qui, étant globalement plus toxiques pour l'hôte que pour le parasite, ne peuvent que perturber de façon favorable les équilibres entre les 2 espèces. Par contre l'imazalil (forte toxicité directe pour les adultes), la triforine (action sur les stades préimaginaux, risque de toxicité pour 
TABLEAU 5

Toxicité pour les populations d'E. formosa et de T. vaporariorum.

Toxicity to $\mathrm{E}$. formosa and $\mathrm{T}$. vaporariorum populations.

\begin{tabular}{|c|c|c|c|c|c|c|c|}
\hline \multirow{2}{*}{ Matic̀res actives } & \multicolumn{3}{|c|}{$\begin{array}{l}\text { \% réduction }\left({ }^{*}\right) \\
T . \text { vaporariorum }\end{array}$} & \multicolumn{3}{|c|}{$\begin{array}{c}\% \text { réduction }\left({ }^{* *}\right) \\
\text { E. formosa }\end{array}$} & \multirow{2}{*}{$\begin{array}{c}\text { Incidences } \\
\text { sur l'équilibre } \\
\text { hôtc-parasitc }\left({ }^{* *}\right)\end{array}$} \\
\hline & $2 \mathrm{CE}$ & $\mathrm{CE}$ & $\frac{\mathrm{CE}}{2}$ & $2 \mathrm{CE}$ & $\mathrm{CE}$ & $\frac{C E}{2}$ & \\
\hline bénomyl & 10,2 & 3,9 & 1,2 & 0,1 & 0 & 0 & 0 \\
\hline bupirimate & 0 & 0 & 0 & 0,2 & 0 & 0 & 0 \\
\hline captafol & 9,0 & 0,1 & 0 & 0 & 0 & 0 & 0 \\
\hline chinométhionate & 63,8 & 54,6 & 45,2 & 22,8 & 16,7 & 12,5 & + \\
\hline dichlofluanide & 0 & 0 & 0 & 0,5 & 0,1 & 0 & 0 \\
\hline folpel & 1,1 & 0,2 & 0,1 & 0 & 0 & 0 & 0 \\
\hline imazalil & 8,8 & 0,1 & 0 & 38,9 & 31,3 & 26,7 & - \\
\hline iprodione & 11,7 & 8,0 & 5,3 & 0 & 0 & 0 & 0 \\
\hline mancozèbc & 51,5 & 43,2 & 35,1 & 8,5 & 5,0 & 3,0 & + \\
\hline pyrazophos & 10,5 & 6,1 & 3,3 & 89,8 & 82,2 & 72.3 & -- \\
\hline thirame & 0 & 0 & 0 & 2,9 & 0,6 & 0,1 & 0 \\
\hline triforine & 32,0 & 24,5 & 18,1 & 45,9 & 37,5 & 31,7 & - \\
\hline vinchlozoline & 0 & 0 & 0 & 1,4 & 0,3 & 0,1 & 0 \\
\hline cyhcxatin & 73,8 & 64,3 & 53,7 & 29,4 & 16,4 & 8,0 & + \\
\hline bioresméthrine & 81,1 & 60,1 & 35,8 & 72,1 & 65,2 & 58,3 & 0 \\
\hline deltaméthrine & 95,7 & 92,7 & 88,5 & 99,9 & 97,9 & 87,3 & -- \\
\hline dichlorvos & 67,1 & 62,7 & 58,0 & 69,6 & 60,6 & 52,3 & 0 \\
\hline parathion-éthyl & 91,5 & 86,7 & 80,4 & 100 & 100 & 99,9 & -- \\
\hline pyrimicarbe & 0 & 0 & 0 & 45,7 & 43,6 & 40,1 & - \\
\hline pyrimiphos-méthyl & 78,1 & 66,5 & 53,1 & 99,8 & 99,2 & 97,3 & -- \\
\hline
\end{tabular}

$\left(^{*}\right)$ Réduction des populations d'aleurodes observées par rapport au témoin.

$\left({ }^{* *}\right)$ Impact global du traitement sur les populations d'E. formosa (réductions d'émergence des stades préimaginaux + toxicité directe et rémanence sur adultes).

$\left({ }^{* * *}\right)+$ : favorable $; 0$ : sans effets notables $;-$ : défavorable $;--:$ très défavorable.

les adultes) et le pyrimicarbe (forte toxicité pour les adultes), bien qu'ayant un impact global assez faible (inférieur à 50 p. 100), sont à utiliser avec prudence car ils entraînent un déséquilibre défavorable au parasite, surtout dans l'hypothèse de traitements répétés. Enfin le pyrazophos, la deltaméthrine, le parathion-éthyl et le pyrimiphosméthyl, très toxiques pour tous les stades d' $E$. formosa et très persistants, sont à proscrire formellement dans le cadre d'une lutte intégrée.

\section{DISCUSSION ET CONCLUSIONS}

L'étude des facteurs de toxicité des 20 pesticides testés permet de tirer les conclusions générales suivantes: s'il semble possible de trouver des fongicides n'interférant pas avec la lutte biologique utilisant $E$. formosa, cela semble beaucoup plus difficile pour les insecticides : au mieux, dans ce cas, pouvons-nous trouver des produits affectant plutôt l'hôte que le parasite. D'une manière générale, c'est le stade adulte qui est le plus sensible, touché par les traitements directs ou par les résidus présents sur les plantes : c'est ce dernier facteur qui est certainement le plus important dans le choix des insecticides; on peut en effet constater que les produits ayant une longue persistance d'action (pyrazophos, deltaméthrine, parathion-éthyl et pyrimiphos-méthyl) sont globalement les plus toxiques et perturbent le plus les équilibres hôtes-parasites.

Les stades pré-imaginaux sont généralement moins sensibles et constituent la phase de tolérance vis-à-vis des pesticides. Cette tolérance n'est malheureusement pas absolue: les jeunes stades internes aux larves d'aleurodes peuvent être touchés soit directement (pyrazophos) soit indirectement, la mort de l'hôte entraînant obligatoirement la mort du parasite ; après noircissement, la tolérance est plus grande, l'hôte déjà mort assurant par son tégument une certaine protection.

D'un point de vue pratique sont utilisables sans réserves comme anti-oïdiums, le bupirimate et la dichlofluanide ; l'imazalyl pourrait être utilisé dans la mesure ou peu d'adultes sont présents au moment du traitement et immédiatement après. Le cas du chinométhionate est particulier : il présente un certain risque de toxicité pour les adultes mais il est efficace contre les aleurodes; de notre point de vue, c'est donc un bon produit pour la lutte intégrée et cela confirme les expérimentations de MC ClanAHAN (1970) et de Elenkov et al. (1975). Par contre Ledieu (1979), à la suite d'expérimentations en serre, le proscrit, des traitements successifs à 1 semaine d'écart éliminant totalement les 2 populations hôte et parasite.

Comme anti-Botrytis, le bénomyl, la dichlofluanide, l'iprodione, le thirame et la vinchlozoline ne présentent aucune toxicité ; le choix devra donc se faire uniquement sur l'efficacité vis-à-vis du champignon.

Nous disposons comme anti-mildiou du captafol, de la dichlofluanide, du folpel et du mancozèbe, ce dernier favorisant $E$. formosa.

La maladie fongique contre laquelle nous sommes le plus démunis est probablement la cladosporiose de la tomate ; la triforine, seul produit efficace en traitement curatif, est malheureusement relativement toxique pour $E$. formosa et ce pour la plupart des stades; des traitements répétitifs avec ce produit risquent en quelques semaines de détruire complètement les populations du parasite. 
La lutte chimique contre les acariens semble facile à résoudre dans le cadre de la lutte intégrée : le cyhexatin s'est révélé plus toxique pour l'aleurode que pour son parasite et le chinométhionate dont nous avons parlé plus haut est également un bon acaricide. L'association classique dicofol-tétradifon testée par LEDIEU (1979) semble également utilisable. De plus, nous disposons également de moyens biologiques de lutte, en particulier $\boldsymbol{P}$. persimilis, utilisables contre les tétranyques.

La lutte chimique contre les insectes ravageurs pose un problème plus difficile à résoudre. La compatibilité des insecticides avec $E$. formosa est en effet difficile du fait de la grande sensibilité des adultes : ainsi le pyrimicarbe, qui est toujours mentionné comme aphicide spécifique, exerce visà-vis des adultes d'E. formosa une toxicité non négligeable ; pratiquement tous les parasites adultes présents lors d'un traitement sont détruits et les effets toxiques des résidus persistent au moins une semaine. Ceci confirme le travail de IRVING \& WYATT (1973). Heureusement nous avons montré que cet aphicide n'est pas toxique pour les stades préimaginaux, observation faite également par PARR \& BinNS (1970) qui laisse certaines possibilités de programmation dans un schéma de lutte intégrée.

La lutte vis-à-vis des autres ravageurs occasionnels ou secondaires des cultures protégées passe obligatoirement par l'utilisation d'insecticides peu persistants et efficaces contre les aleurodes comme par exemple la bioresméthrine ou le dichlorvos. Il faut cependant veiller à les utiliser avec précaution (KowALSKA et al. 1980) soit préventivement ou en présence de peu d'adultes, soit en traitements localisés sur les parties des plantes où les adultes d'aleurodes sont nombreux et les parasites absents, ce qui correspond aux 5-6 plus jeunes feuilles de la culture.

Outre l'aspect pratique de choix des produits en vue de leur utilisation dans des schémas de lutte intégrée, ce travail montre la complexité des facteurs mis en cause dans l'expression de la toxicité d'un produit : sensibilité différentielle des stades, caractéristiques propres du pesticide (persistance) et importance de la toxicité vis-à-vis de l'hôte pour comprendre l'impact réel du produit, lorsque comme ici, un parasite spécifique évolue dans un milieu clos.

Ceci montre la difficulté d'élaborer des méthodes standardisées à la fois fiables et simples telles celles dont la mise au point est étudiée dans le cadre du groupe de travail de l'OILB, "Pesticides and Beneficial Arthropods".

Il est nécessaire d'y associer des essais complémentaires visant à préciser certains aspects de la toxicité des pesticides, tels ceux étudiés ci-dessus, mais aussi, par exemple, des méthodologies d'ćtude de l'effet répulsif des produits.

Reçu le 24 décembre 1982. Accepté le 21 février 1983.

\section{RÉFÉRENCES BIBLIOGRAPHIQUES}

Di Pietro J. P., 1977. Contribution à l'étude d'une méthodologie de lutte biologique contre l'aleurode des serres, Trialeurodes vaporariorum Westw. (Homoptera, Aleurodidae) Thèse Doct. Spécialité, Fac. Sc. Toulouse, $112 \mathrm{p}$.

Elenkov E., Khristova E., Shanab L. M., Spasova P., 1975. Toxicity of some fungicides against the greenhouse whitefly Trialeurodes vaporariorum West. and its parasite Encarsia formosa Gah. Acta Phytopathol. Acad. Sci. hung., 10, 165-176.

Gahan A. B., 1924. Some new parasitic hymenoptera with notes on several described forms. Proc. U.S. Nai. Mus., 61, 1-23.

Helgesen R. G., Tauber M. J., 1974. Pirimicarb, an aphicide non toxic to three entomophagous arthropods. Environ. Entomol., 3, 99-101.

lacob N., Posoiu V., Manolescu H., 198.1. Sélectivité de quclques pesticides vis-à-vis des principaux entomophages utilisés en luttc intégrée dans les serres (en roumain). An. Inst. Cercet Prot. Plant., 16, 357-373.

Irving S. N., Wyatt I. J., 1973. Effect of sublethal doses of pesticides on the oviposition behaviour of Encarsia formosa. Ann. appl. Biol., 75, 57-62.
Kowalska T., Szczepanska K., Bartkowiak A., 1980. Studies on pesticides effect on Trialeurodes vaporariwrum and its parasite Encarsia formosa. Bull. SROP., 3, 101-112

Ledieu M. S., 1979. Laboratory and glasshouse screening of pesticides for adverse effects on the parasite Encarsia formosa Gahan. Pestic. Sci., 10, 123-132.

Mc Clanahan R. J., 1970. Integrated control of the greenhouse whitefly on cucumbers. $J$. econ. Entomol., 63, 599-601.

Onillon J. C., 1982. L'alcurode des serres Trialeurodes vaporariorum Westwood ct son contrólc biologique. Def. Veg., 36. 59-63.

Parr W. J., Binns E. S., 1970. Effect of chemicals on Encarsia. Glasshouse Crops Res. Inst. annu. Rep. 1969, 112.

Van Alphen J. J. M., Nell H. W., Sevenster-Van der Lelie L. A., 1976. The parasite host relationship between Encarsia formosa G. (Hym., Aphelinidae) and Trialeurodes vaporariorum Westw. (Homopt., Aleurodidae). The importance of hostfeeding as a mortality factor in greenhouse whitefly nymphs. Bull. OILB/SROP "Lutte intégrée en cultures sous serres", 1976\%/4, 165-169. 